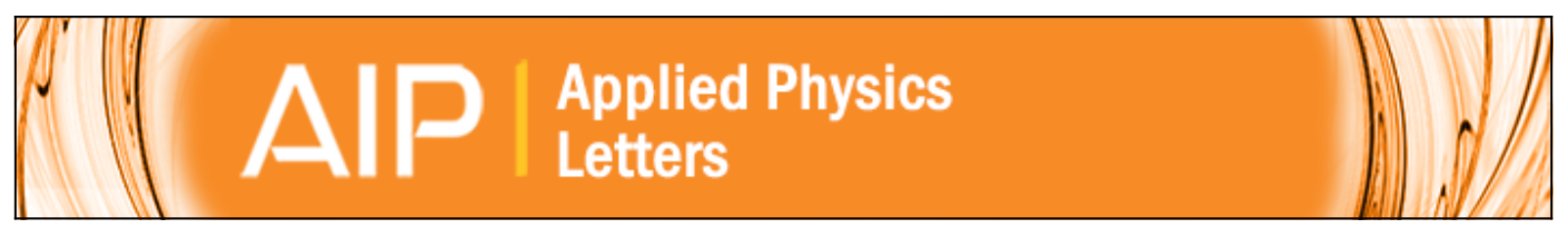

Genetically designed L3 photonic crystal nanocavities with measured quality factor exceeding one million

Y. Lai, S. Pirotta, G. Urbinati, D. Gerace, M. Minkov, V. Savona, A. Badolato, and M. Galli

Citation: Applied Physics Letters 104, 241101 (2014); doi: 10.1063/1.4882860

View online: http://dx.doi.org/10.1063/1.4882860

View Table of Contents: http://scitation.aip.org/content/aip/journal/apl/104/24?ver=pdfcov

Published by the AIP Publishing

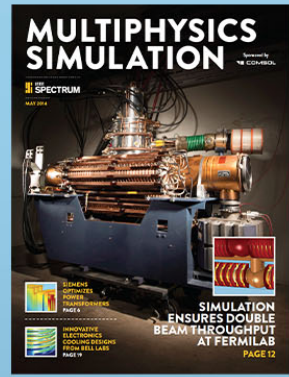

Free online magazine

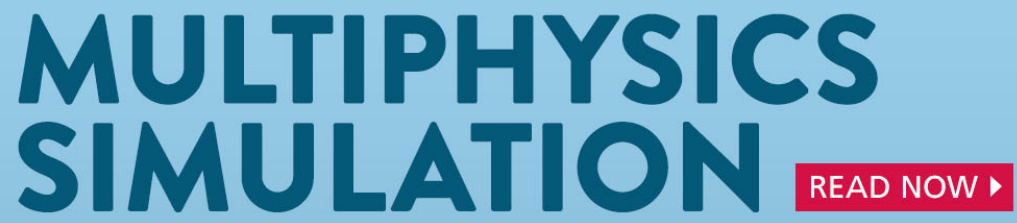




\title{
Genetically designed L3 photonic crystal nanocavities with measured quality factor exceeding one million
}

\author{
Y. Lai, ${ }^{1}$ S. Pirotta, ${ }^{2}$ G. Urbinati, ${ }^{2}$ D. Gerace,${ }^{2}$ M. Minkov,${ }^{3}$ V. Savona,${ }^{3}$ A. Badolato, ${ }^{1, a)}$ \\ and M. Galli ${ }^{2}$ \\ ${ }^{1}$ Department of Physics and Astronomy, University of Rochester, Rochester, New York 14627, USA \\ ${ }^{2}$ Dipartimento di Fisica, Università di Pavia, via Bassi 6, 27100 Pavia, Italy \\ ${ }^{3}$ Laboratory of Theoretical Physics of Nanosystems, Ecole Polytechnique Federale de Lausanne EPFL, \\ CH-1015 Lausanne, Switzerland
}

(Received 8 March 2014; accepted 9 April 2014; published online 16 June 2014)

\begin{abstract}
We report on the experimental realization of ultra-high quality factor $(Q)$ designs of the L3-type photonic crystal nanocavity. Based on genetic optimization of the positions of few nearby holes, our design drastically improves the performance of the conventional L3 as experimentally confirmed by direct measurement of $Q \simeq 2 \times 10^{6}$ in a silicon-based photonic crystal membrane. Our devices rank among the highest $Q / V$ ratios ever reported in photonic crystal cavities, holding great promise for the realization of integrated photonic platforms based on ultra-high- $Q$ resonators. (C) 2014 AIP Publishing LLC. [http://dx.doi.org/10.1063/1.4882860]
\end{abstract}

Photonic crystal nanocavities $(\mathrm{PCNs})^{1}$ are becoming the leading paradigm for ultra-integrated nanophotonics, due to their ability to confine light in small spaces for long times. Thanks to such a confinement, light-matter interaction can be strongly enhanced and ultimately reach the regime of cavity quantum electrodynamics. ${ }^{2}$ Over the past decade, progress in nanofabrication and modeling has brought the main attention on few PCN designs featuring measured quality factors $(Q)$ approaching the $10^{7}$ range and/or ultra-small mode volumes $(V) .^{3-7}$ Nonetheless, the simplest and most widespread designs (the L3, H0, and $\mathrm{H} 1)^{8,9}$ had been regarded as moderate- $Q$ PCNs with both theoretical and measured $Q$-values never exceeding $0.3 \times 10^{6}$. Very recently, this belief has been challenged by a shift of paradigm in PCN modeling. ${ }^{10}$

Here, we demonstrate that the L3-type PCN can be drastically improved by combining a genetic optimization tool with a fast simulation algorithm based on the guided mode expansion (GME) method. ${ }^{11} \mathrm{We}$ report on the experimental realization of such a super-L3 PCN fabricated in a silicon membrane. In contrast to the conventional L3, our cavity features measured $Q$-values systematically exceeding $10^{6}$ while maintaining a very small footprint and ultra-small $V$. Our experiment prototypes de facto a transformative generation of PCNs.

By letting the genetic optimization procedure vary only the positions of five holes next to the defect, we determined the design that maximizes the $Q$. The following parameters were used in the simulations: silicon refractive index $n=3.46$, silicon slab thickness $d=220 \mathrm{~nm}$, PCN lattice period $a=400 \mathrm{~nm}$, and hole radius $r=0.25 a$. A schematic of the design is shown in Fig. 1(a). Five nearest edge holes are outward shifted along the cavity axis ( $\mathrm{K}$ symmetry direction of the triangular lattice) by $\mathrm{S}_{1}=0.3370 a, \mathrm{~S}_{2}=0.2706 a$, $\mathrm{S}_{3}=0.0874 a, \mathrm{~S}_{4}=0.3223 a, \mathrm{~S}_{5}=0.1737 a$, symmetrically on both sides of the cavity. Such a design results in a theoretical

\footnotetext{
a) Author to whom correspondence should be addressed. Email: antonio.badolato@gmail.com
}

GME-computed $Q_{\mathrm{th}}=5.0 \times 10^{6}$ at the resonant wavelength $\lambda=1555 \mathrm{~nm}$. Our Finite-Difference Time-Domain (FDTD) simulations substantially confirmed the value of $\lambda$ and $Q_{\mathrm{th}}$, and resulted in a mode volume ${ }^{12} V=0.96(\lambda / n)^{3}$ - still significantly smaller than the values, ranging from 1.2 to $2.0(\lambda / n)^{3}$, that characterize the current highest- $Q$ designs. ${ }^{3,4}$ Compared to the previously reported L3 PCNs theoretical values $\left(Q_{\mathrm{th}}=0.26 \times 10^{6}, V=0.73(\lambda / n)^{3}\right.$ in Ref. 13), our $Q_{\mathrm{th}} / V$ is roughly 15 times larger.

To validate our design, we fabricated eight groups of super-L3 PCNs in commercially available silicon on insulator (SOI) wafers. Each group comprised twenty-one nominally identical PCNs, while the hole radius was slightly varied from one group to the next, resulting in a different nominal resonant wavelength for each group. By varying only the radius instead of applying an overall scaling factor, the design moves away from its optimal configuration. In

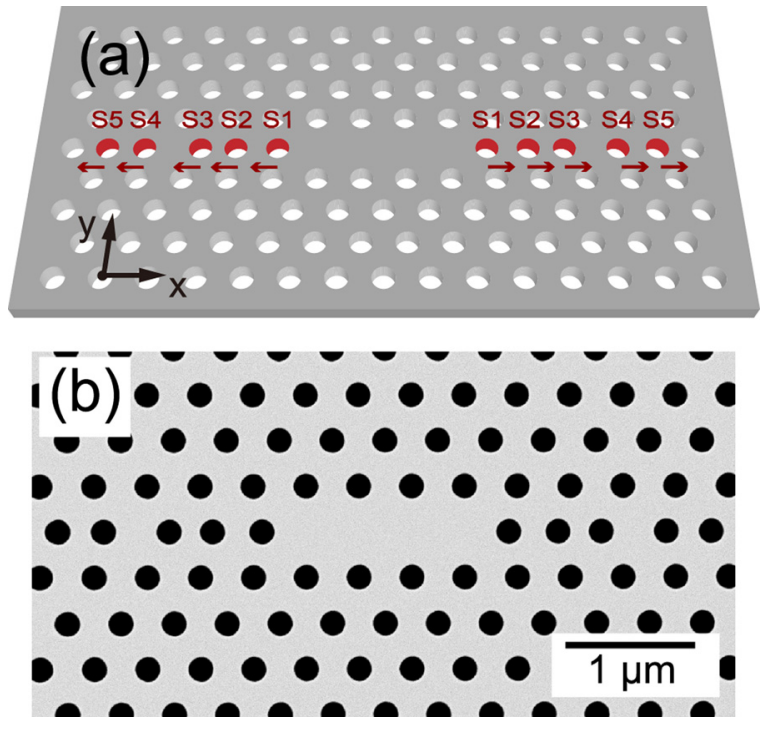

FIG. 1. (a) Design of the super-L3 PCN. The positions of five nearest-edge holes are outward-shifted along the $\mathrm{X}$-direction by amounts denoted by $\mathrm{S}_{1}$, $\mathrm{S}_{2}, \mathrm{~S}_{3}, \mathrm{~S}_{4}, \mathrm{~S}_{5}$. (b) Scanning-electron-microscope top-view image of a fabricated super-L3 PCN. 
particular, with our choice of fabrication parameters, the group number five was the one nominally corresponding to the optimal design.

The SOI wafer consisted of a $220 \mathrm{~nm}$ top silicon layer and a $3 \mu \mathrm{m}$ buffer oxide layer on a silicon substrate. The PCN pattern was defined by $100 \mathrm{kV}$ electron beam lithography direct writing using positive e-beam resist. The pattern was transferred from the resist to the silicon top-layer by fluorine based inductively coupled plasma dry etching and the oxide layer was removed by dilute HF wet etching. Engineered lateral openings in the membrane made the suspended PCN free from buckling. ${ }^{14}$ Fig. 1(b) shows the scanning electron microscope image of a super-L3 PCN whereby we measured the standard deviation of the hole-radii to be $<1 \mathrm{~nm}$.

The fundamental PCN mode was measured by crosspolarization resonant scattering (RS) spectroscopy. ${ }^{15}$ This technique is particularly suited for measuring PCNs with ultra-high $Q$ s, because it does not require any evanescent coupling (e.g., with an integrated photonic crystal waveguide or an external fiber taper) and thus directly yields the intrinsic (unloaded) $Q$. A schematic of the experimental setup is shown in Fig. 2(a), where a $10 \mathrm{MHz}$ resolution tunable laser was used to excite resonantly the cavity mode, and the light scattered by the cavity was collected vertically in far-field with a NA $=0.9$ objective. In this setting, the emission of the fundamental mode resulted to be linearly polarized (y-direction). Using the y-direction as reference, we excited with a laser beam linearly polarized at $+45^{\circ}$ and collected at $-45^{\circ}$ achieving precise control over the extinction of the reflected
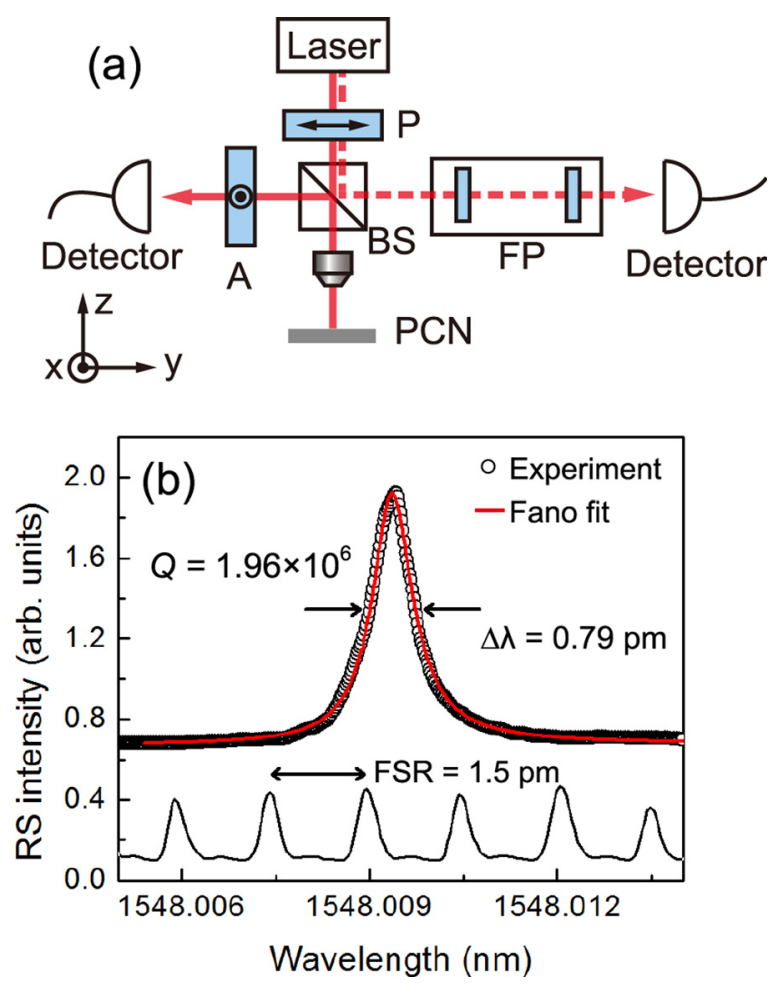

FIG. 2. (a) Schematic of the cross-polarization resonant scattering setup. P: polarizer, A: polarization analyzer, BS: beam splitter, FP: Fabry-Pérot interferometer. (b) Resonant scattering spectrum of the super-L3 PCN (open dots). Best-fitted Fano lineshape (red line) gives a linewidth of $0.79 \mathrm{pm}$, i.e., $Q=1.96 \times 10^{6}$. The Fabry-Pérot spectrum used for wavelength calibration is shown as a black line at the bottom of the plot. laser light. While scanning, the laser wavelength was calibrated through the simultaneous measurement of the interference fringes of a $0.8 \mathrm{~m}$ long Fabry-Pérot interferometer with $1.5 \mathrm{pm}$ free spectral range. Fig. 2(b) shows the RS spectrum of our super-L3 PCN fundamental mode measured on the cavity displaying the highest $Q$ out of the twenty-one PCNs in group five. The lower curve in Fig. 2(b) shows the measured transmission spectrum of the Fabry-Pérot interferometer. Fitting of the cavity resonance with a Fanolineshape $^{15}$ demonstrates $Q_{\exp }=1.96 \times 10^{6}$, which is 20 times larger than the highest $Q$ previously reported on a L3 PCNs. ${ }^{13,16}$ This corresponds to an experimentally observed $Q_{\text {exp }} / V>2 \times 10^{6}(\lambda / n)^{-3}$, which is more than 10 times larger than the values previously reported in L3 PCNs and ranks our super-L3 among the highest $Q_{\exp } / V$ ever reported in 2D PCNs.

The other group-five-PCNs all displayed a clear resonance, with $Q$-values lying systematically in the $1 \times 10^{6}$ range, as plotted in Fig. 3. Spectra measured from devices belonging to groups other than group-five (data not shown) resulted in $Q$-values ranging from $0.4 \times 10^{6}$ to $1.1 \times 10^{6}$. This finding is consistent with group-five-PCNs being the ones closest to the nominally optimal design. We notice that the $Q$-values measured in the non-optimal groups-all largely exceeding the highest $Q$-values previously measured for a $\mathrm{L} 3 \mathrm{PCN}^{13}$-also demonstrate the robustness of the present design to small variations of the hole radius.

Fluctuations in the values of $Q$ and $\lambda$ (Fig. 3) are due to intrinsic disorder introduced by the fabrication process. In Fig. 4, we report the occurrence histograms of the values of $Q$ and $\lambda$ measured on the group-five-PCNs. In the same panels, we plot the theoretical occurrence histograms obtained by simulating with GME the optimal design. Disorder was modeled by adding a random, zero-mean, Gauss-distributed offset to the nominal radius and position of each circular hole, assuming a standard deviation $\sigma .{ }^{17,18}$ It was previously shown that this simple model correctly describes the effect of a more realistic disorder pattern in which holes may be irregularly shaped. ${ }^{17}$ Denoting with $Q_{\mathrm{d}}{ }^{-1}$ the additional loss rate induced by disorder, and with $Q_{\mathrm{a}}^{-1}$ the effective constant loss rate due to background absorption, the total value of $Q$ predicted

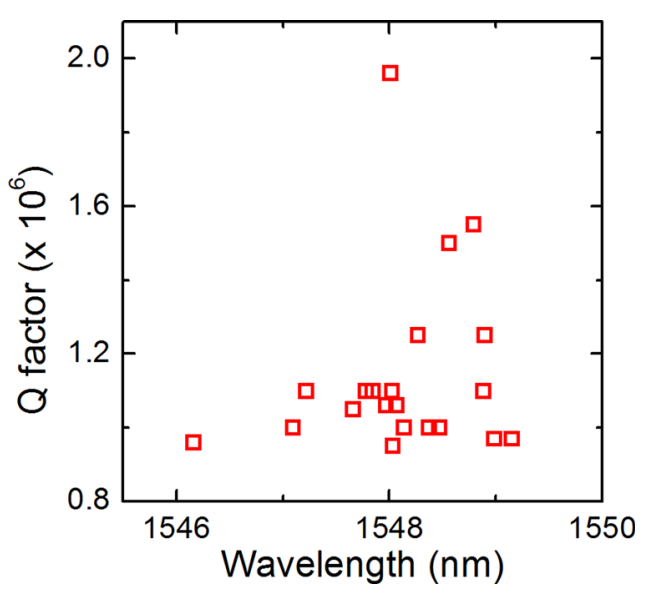

FIG. 3. Scatter plot of the measured $Q$-values and resonant wavelengths from the twenty-one nominally identical PCNs present in group five. All PCNs display clear resonance with $Q$-values lying in the $1 \times 10^{6}$ range. The highest value (spectrum in Fig. 2(b)) corresponds to $Q=1.96 \times 10^{6}$. 

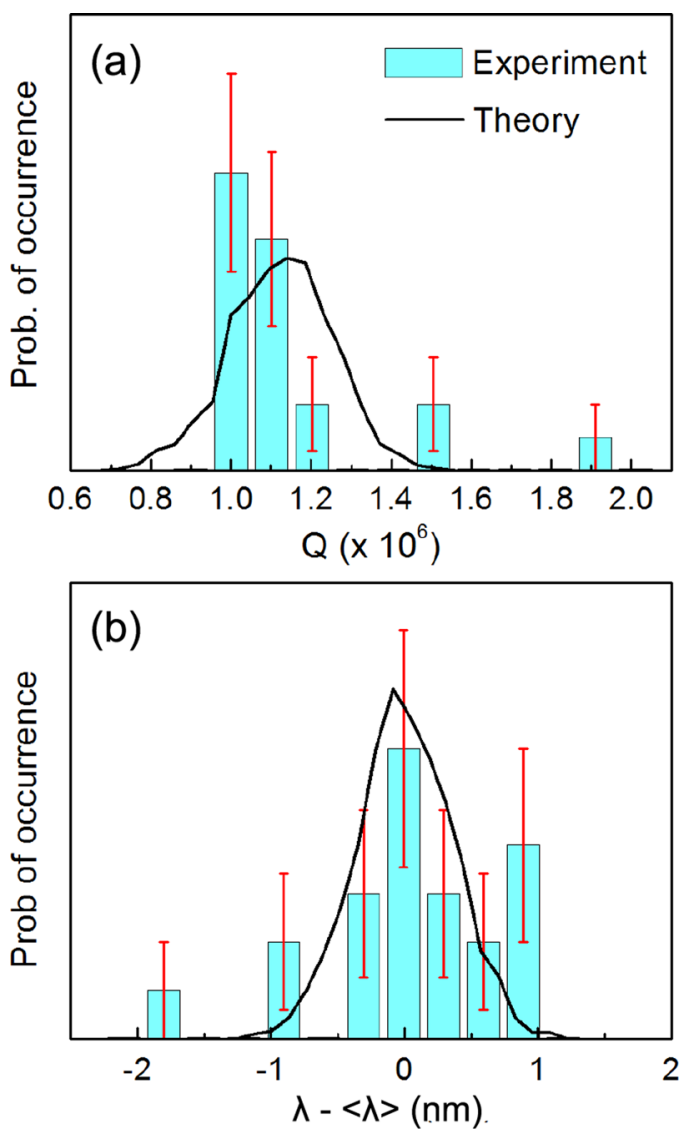

FIG. 4. Occurrence histograms of (a) $Q$-values and (b) resonant wavelengths, as measured in group five. In both panels, the black lines denote the histogram computed over 1500 simulated realizations of the super-L3 PCN design with disorder added as Gaussian fluctuations of the hole radii and positions. The red error bars indicate the statistical uncertainty in the experimental data.

by the theory is $Q=\left(Q_{\mathrm{th}}{ }^{-1}+Q_{\mathrm{d}}{ }^{-1}+Q_{\mathrm{a}}{ }^{-1}\right)^{-1}$, where $Q_{\mathrm{th}}$ is the value simulated in the absence of disorder. The values of $\sigma$ and $Q_{\mathrm{a}}$ have been empirically adjusted to $\sigma=0.0014 \mathrm{a}$, corresponding to $\left(\left\langle 1 / Q_{\mathrm{d}}\right\rangle\right)^{-1}=4.5 \times 10^{6}$, and $Q_{\mathrm{a}}=2.1 \times 10^{6}$ to reproduce at best the measured histograms. A fairly good agreement is found, although the measured $Q$ seems to obey a narrower distribution than the simulated one. We argue that this discrepancy might be due partly to the limited number of experimental data, resulting in large statistical uncertainty, and partly to the simple disorder model, which neglects possible spatial correlations in the fluctuations of different holes. Moreover, additional aspects, such as slight surface oxidation of the silicon membrane upon air exposure as well as surface contamination by adsorption ${ }^{7}$ of water vapor molecules, may also affect the influence of disorder on the measured $Q$.

As a further proof of the general extent of our framework, we have repeated the genetic design by varying only the first three-hole-shifts $S_{1}, S_{2}$, and $S_{3}$ of the L3. We obtained an optimal design with a GME-computed $Q_{\text {th }}=2.1 \times 10^{6}$ and a FDTD-computed $V=0.94(\lambda / n)^{3}$, for the values $S_{1}=0.3266 a, S_{2}=0.2577 a$, and $S_{3}=0.1162 a$. By employing the same variational parameters, such a design improves on a previous optimal one ${ }^{13}$ by almost one order of magnitude while reducing even more the device footprint. PCNs with such a three-hole-shift design gave experimental $Q$-values as high as $Q_{\exp }=0.88 \times 10^{6}$.
In a PCN, radiation losses are reduced by minimizing the amplitude of the Fourier components of the mode that lie inside the light cone. Previous works have achieved this goal by imposing a specific mode shape in reciprocal space. ${ }^{19}$ In such case, the corresponding PCN design was determined through an inverse-problem approach relying on a semianalytical approximation. Here, the same goal is achieved through an essentially exhaustive search within the parameter space, based on a virtually first-principle simulation method such as the GME. Thus, for a given set of variational parameters, our method can arguably explore high- $Q$ configurations that may be overlooked by alternative approximate methods. On the other hand, the (acceptable) drawback of this approach is the lack of an intuitive physical understanding of the mechanism minimizing radiation losses in the optimal configurations.

In conclusion, we presented a transformative generation of L3 PCNs, which features ultra-high $Q / V$ while maintaining very small footprint and high fabrication yield. The PCN designs were realized through a genetic optimization protocol that enables systematic improvement of the $Q$-values and our experiments confirmed the drastic improvement brought by the optimized design. The same protocol might in the future bring to a major advance in the design of several photonic crystal structures, among which cavities based on a silicon-on-insulator photonic crystal, $^{20,21}$ cavities with simultaneous first- and second-harmonic resonances, ${ }^{22,23}$ and hollow cavities for optical trapping and sensing. ${ }^{24} \mathrm{We}$ emphasize the relevance of our super-L3 PCN as a highly promising building block for integrated photonics applications including silicon photonics, on-chip scalable platforms for quantum state control, ${ }^{2}$ and cavity optomechanics. ${ }^{25}$

Note added in proof: Very recently, an experimental study ${ }^{26}$ of silicon L3 PCNs, optimized using an educated-guess procedure, reported a measured $Q$-factor of $10^{6}$ for the best cavity (and a value $Q=0.5 \times 10^{6}$ averaged on several PCNs). Our result still outperforms these values by a factor of 2 .

Y.L. and A.B. acknowledge partial support from National Science Foundation (NSF) under Grant No. DMR1309734. Fabrication was performed at the Cornell NanoScale Science \& Technology Facility partially supported by NSF. M.M. and V.S. acknowledge support by the Swiss National Science Foundation (SNSF) through Project No. 200020_132407. D.G. and V.S. acknowledge the SNSF for support through the International Short Visits program, Project No. IZK0Z2-150900.

${ }^{1}$ M. Notomi, Rep. Prog. Phys. 73, 096501 (2010).

${ }^{2}$ T. Volz, A. Reinhard, M. Winger, A. Badolato, K. J. Hennessy, E. L. Hu, and A. Imamoğlu, Nat. Photonics 6, 607 (2012).

${ }^{3}$ B.-S. Song, S. Noda, T. Asano, and Y. Akahane, Nat. Mater. 4, 207 (2005).

${ }^{4}$ E. Kuramochi, M. Notomi, S. Mitsugi, A. Shinya, T. Tanabe, and T. Watanabe, Appl. Phys. Lett. 88, 041112 (2006).

${ }^{5}$ Z. Han, X. Checoury, D. Néel, S. David, M. El Kurdi, and P. Boucaud, Opt. Commun. 283, 4387 (2010).

${ }^{6}$ Y. Taguchi, Y. Takahashi, Y. Sato, T. Asano, and S. Noda, Opt. Express 19, 11916 (2011).

${ }^{7}$ H. Sekoguchi, Y. Takahashi, T. Asano, and S. Noda, Opt. Express 22, 916 (2014). 
${ }^{8}$ Y. Akahane, T. Asano, B.-S. Song, and S. Noda, Nature 425, 944 (2003). ${ }^{9}$ O. Painter, R. K. Lee, A. Scherer, A. Yariv, J. D. O’Brien, P. D. Dapkus, and I. Kim, Science 284, 1819 (1999).

${ }^{10}$ U. P. Dharanipathy, M. Minkov, M. Tonin, V. Savona, and R. Houdré, "Evolutionarily optimized ultrahigh-Q photonic crystal nanocavity," e-print arXiv:1311.0997v1.

${ }^{11}$ L. C. Andreani and D. Gerace, Phys. Rev. B 73, 235114 (2006).

${ }^{12} \mathrm{We}$ adopted the most accepted definition of effective mode volume for our PCN; see Refs. 1, 10, and 13.

${ }^{13}$ Y. Akahane, T. Asano, B.-S. Song, and S. Noda, Opt. Express 13, 1202 (2005).

${ }^{14}$ E. Iwase, P.-C. Hui, D. Woolf, A. W. Rodriguez, S. G. Johnson, F. Capasso, and M. Lončar, J. Micromech. Microeng. 22, 065028 (2012).

${ }^{15}$ M. Galli, S. L. Portalupi, M. Belotti, L. C. Andreani, L. O'Faolain, and T. F. Krauss, Appl. Phys. Lett. 94, 071101 (2009).

${ }^{16}$ S. L. Portalupi, M. Galli, M. Belotti, L. C. Andreani, T. F. Krauss, and L. O'Faolain, Phys. Rev. B 84, 045423 (2011).

${ }^{17}$ M. Minkov and V. Savona, Opt. Lett. 37, 3108 (2012).
${ }^{18}$ M. Minkov, U. P. Dharanipathy, R. Houdré, and V. Savona, Opt. Express 21, 28233 (2013).

${ }^{19}$ D. Englund, I. Fushman, and J. Vučković, Opt. Express 13, 5961 (2005).

${ }^{20}$ Z. Han, X. Checoury, L.-D. Haret, and P. Boucaud, Opt. Lett. 36, 1749 (2011).

${ }^{21}$ Y. Tanaka, T. Asano, R. Hatsuta, and S. Noda, Appl. Phys. Lett. 88, 011112 (2006).

${ }^{22}$ S. Buckley, M. Radulaski, K. Biermann, and J. Vučković, Appl. Phys. Lett. 103, 211117 (2013).

${ }^{23}$ S. Diziain, R. Geiss, M. Zilk, F. Schrempel, E.-B. Kley, A. Tünnermann, and T. Pertsch, Appl. Phys. Lett. 103, 051117 (2013).

${ }^{24}$ N. Descharmes, U. P. Dharanipathy, Z. Diao, M. Tonin, and R. Houdré, Phys. Rev. Lett. 110, 123601 (2013).

${ }^{25}$ M. Aspelmeyer, T. Kippenberg, and F. Marquardt, "Cavity optomechanics," e-print arXiv:1303.0733 [cond-mat.mes-hall].

${ }^{26}$ E. Kuramochi, K. Nozaki, A. Shinya, K. Takeda, T. Sato, S. Matsuo, H. Taniyama, H. Sumikura, and M. Notomi, Nat. Photonics 8, 474 (2014). 